\title{
Osteoblastoma of the Thyroid Cartilage Treated With Voice Preserving Laryngeal Framework Resection
}

\author{
Tiffany A. Glazer, MD; Matthew E. Spector, MD; Jonathan McHugh, MD; \\ Norman D. Hogikyan, MD
}

\begin{abstract}
Objectives/Hypothesis: Osteoblastoma is a slow-growing, locally destructive benign bone neoplasm, rarely occurring in the laryngeal cartilage. We present the case of a professional voice user diagnosed with laryngeal osteoblastoma after microdirect laryngoscopy and endoscopic biopsy. Her treatment required a unique operation, with elements of partial laryngectomy and maintenance of vital endolaryngeal soft tissues, in order to optimize vocal outcome.
\end{abstract}

Key Words: Early glottic cancer; voice/dysphonia; osteoblastoma; thyroid cartilage.

Laryngoscope, 123:1948-1951, 2013

\section{INTRODUCTION}

Osteoblastoma is a slow-growing, locally destructive benign bone neoplasm, typically occurring in the vertebral column. The incidence of skull and facial involvement, including the cervical spine and temporal, occipital, ethmoid, frontal, sphenoid, and gnathic bones, has been reported as $2 \%$ to $20 \%$ of all cases of osteoblastoma. ${ }^{1}$ Osteoblastoma of the larynx is extremely rare, with only six cases reported in the literature, all of which were in males at least 45 years old. In each case, treatment involved either aggressive resection, such as total laryngectomy in one case and hemilaryngectomy in one case, or other tumor resection procedures that did not spare laryngeal soft tissue. ${ }^{1-6}$ We present the first reported female with osteoblastoma of the thyroid cartilage, treated with en bloc tumor resection but preservation of endolaryngeal soft tissue structures. Osteoblastoma should be distinguished from other bone tumors, such as osteoid osteoma and osteosarcoma, as well as cartilaginous tumors such as chondroma and chondrosarcoma. Tissue biopsy is necessary for definitive diagnosis. Treatment is typically by complete en bloc resection of the lesion.

From the Department of Otolaryngology Head and Neck Surgery (T.A.G., M.E.S., N.D.H.), University of Michigan, Ann Arbor, Michigan, U.S.A., Department of Pathology (J.M.), University of Michigan, Ann Arbor, Michigan, U.S.A.

Editor's Note: This Manuscript was accepted for publication on December 12, 2012.

The authors have no funding, financial relationships, or conflicts of interest to disclose.

Send correspondence to Tiffany A. Glazer, MD, 1500 E. Medical Center Drive, 1904 Taubman Center, Ann Arbor, MI 48109-5132.

E-mail: tglazer@med.umich.edu

DOI: 10.1002/lary.23972

\section{CASE REPORT}

The patient is a 58-year-old woman referred for evaluation in the laryngology clinic after 4 years of worsening dysphonia and throat clearing. Prior to her referral, she had been treated for laryngopharyngeal reflux and possible allergic contributions to her symptoms for years without symptomatic relief. She is a lifetime nonsmoker and nondrinker. She denied dysphagia, odynophagia, otalgia, and hemoptysis. Her voice was moderately hoarse in quality. On laryngeal videostroboscopy, vocal fold mobility was normal bilaterally. There was bruising on the superior surface of the right true vocal fold, with marked submucosal expansion of the fold extending from just below the vibrating margin inferiorly (Fig. 1). Vibratory capacity was severely reduced under stroboscopic light. Computed tomography (CT) scan of the neck demonstrated an $11 \times 14 \mathrm{~mm}$ mixed lytic and sclerotic submucosal lesion, with internal calcifications originating from the right thyroid ala with extension to the left anteriorly (Fig. 2). There was destruction of the inner thyroid cortex with sclerosis and expansion of the adjacent cartilage. The patient was taken to the operating room for microdirect laryngoscopy and endoscopic biopsy through a small lower glottic incision. Intraoperative examination showed marked submucosal expansion beginning just below the free edge of the right true vocal fold (Fig. 3). There was bruising in the supraglottic tissues and herniation of the right ventricular mucosa. There was no mucosal lesion. Palpation of the convex region demonstrated soft tissue overlying a firmer lesion. Biopsies from the submucosal right laryngeal mass showed circumscribed bone that was producing neoplasm, abutting but not destroying the adjacent thyroid cartilage (Fig. 4). At higher power, the lesion was comprised of irregular trabeculae of 


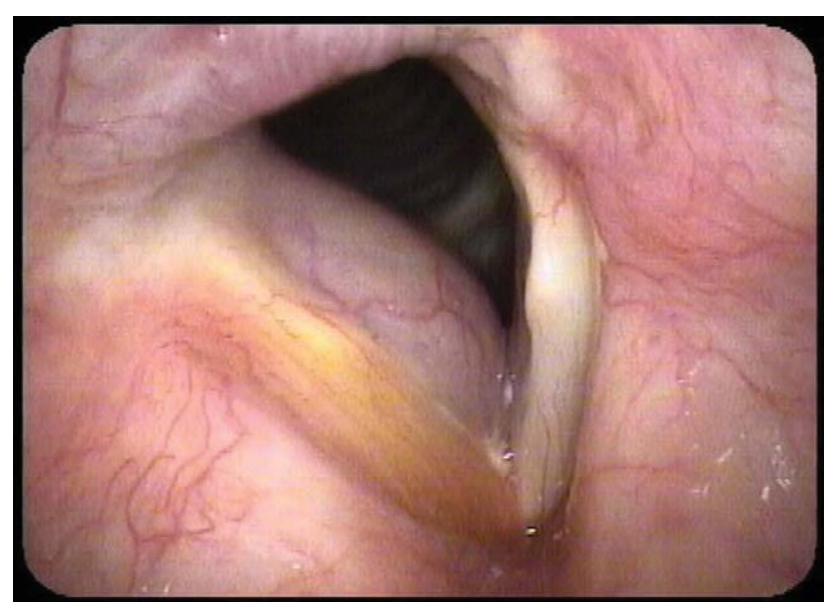

Fig. 1. Still image taken from in-office laryngeal videostroboscopy demonstrating bruising on the superior surface of the right true vocal fold with marked submucosal expansion, extending from just below the vibrating margin inferiorly.

woven bone lined by plump, cytologically bland osteoblasts (Fig. 5). The stroma between the spicules was highly vascular with scattered osteoclastic giant cells.

Since the patient was a professional voice user, optimizing vocal outcome and complete tumor resection were both important surgical priorities. The lesion would require thyroid cartilage resection, but a tailored operation was designed with the intention of sparing internal laryngeal soft tissue and maintaining or reestablishing anterior commissure (AC) attachments if at all possible. She was taken to the operating room where tracheostomy and complete resection were accomplished via an anterior transcervical approach. Internal laryngeal soft tissues were dissected off the thyroid cartilage from above and below the alae, except at the AC, which was left undisturbed. The superior thyroid cartilage cut anteriorly was made above the tumor margin and just inferior to the anterior commissure attachment. The

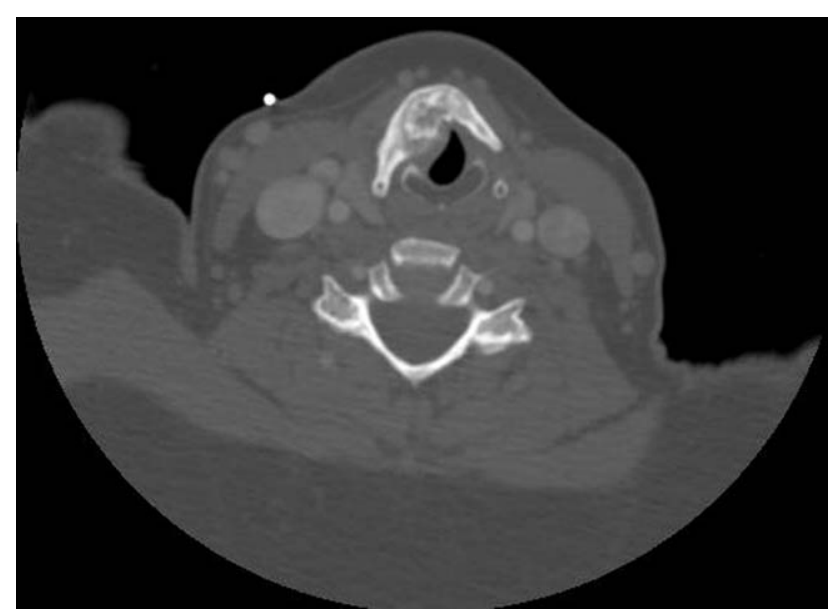

Fig. 2. $1.25 \mathrm{~mm}$ axial slice from computed tomography (CT) scan of the neck with intravenous (IV) contrast, demonstrating an $11 \times$ $14 \mathrm{~mm}$ mixed lytic and sclerotic submucosal lesion, with internal calcifications originating from the right thyroid ala with extension to the left anteriorly.

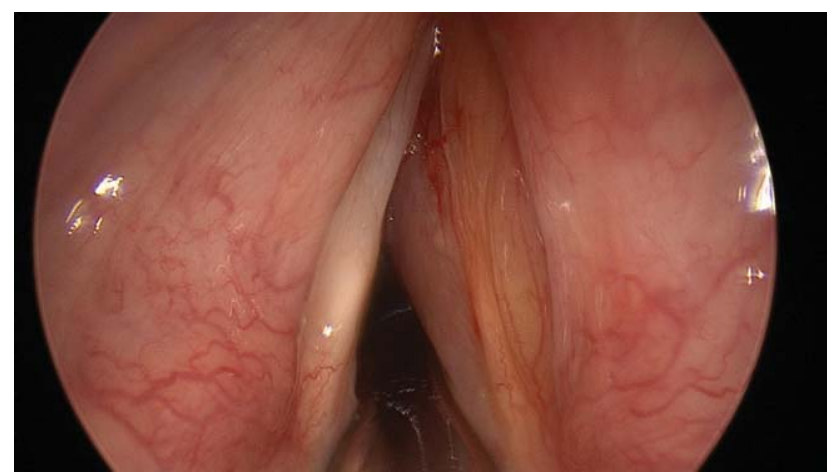

Fig. 3. Microdirect laryngoscopy showed marked right submucosal expansion beginning just below the free edge of the right true vocal fold.

lateral cartilage cuts were determined by the degree of tumor extension on either side. Clear margins were accomplished without resecting any internal laryngeal soft tissue (Fig. 6). The patient had no significant dysphagia postoperatively and was discharged home 4 days later. At 1 week postoperatively she was decannulated. At this appointment, her voice was moderately hoarse, the right vocal fold was edematous, and vocal fold mobility was normal bilaterally (Fig. 7). At 7 weeks postoperatively she had already resumed her professional speaking duties, had mild hoarseness, and the right vocal fold edema was almost completely resolved (Fig. 8).

\section{DISCUSSION}

Osteoblastoma classically presents as localized pain and swelling. Local invasion may produce associated symptoms, such as airway obstruction, dysphonia, or dysphagia if in the larynx. Tumors are typically present for several months or for up to several years before diagnosis. Osteoblastoma can be distinguished from osteoid osteoma clinically, as the latter is rarely progressive, smaller, and typically characterized by sharp, intense

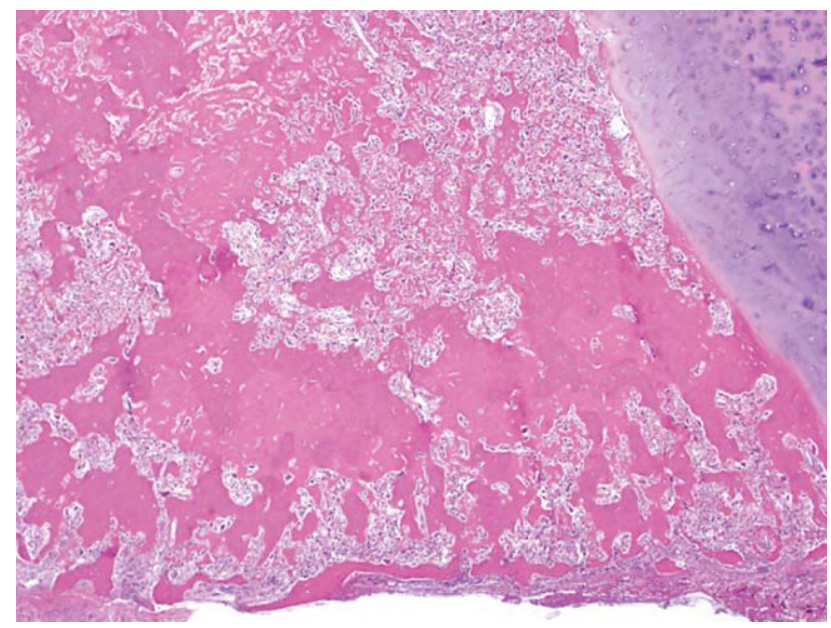

Fig. 4. Biopsy from the submucosal right laryngeal mass showing circumscribed bone-producing neoplasm abutting but not destroying the adjacent thyroid cartilage. 


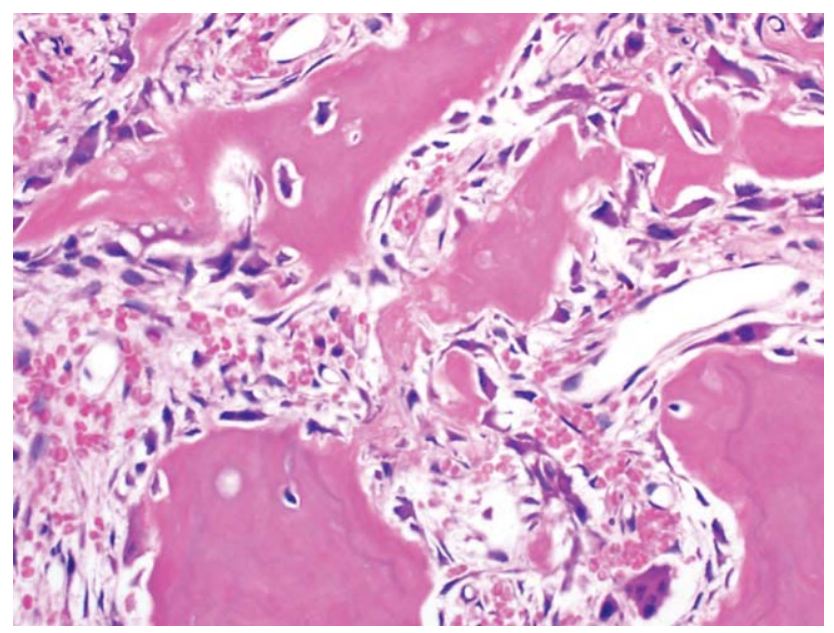

Fig. 5. At higher power, the lesion is comprised of irregular trabeculae of woven bone lined by plump, cytologically bland osteoblasts.

local pain not relieved by nonsteroidal anti-inflammatory drugs (NSAIDs) or aspirin. ${ }^{7,8}$

Plain radiographs may fail to demonstrate osteoblastoma. On CT scan, osteoblastoma typically appears as a well-circumscribed, lucent, expansive defect. Like chondrosarcoma, it is often described with internal calcifications and radiopaque areas. It is often "aggressive" in appearance due to expansion into the surrounding soft tissue. The sometimes aggressive-appearing radiologic appearance makes an osteoblastoma difficult to distinguish from an osteosarcoma based on radiology alone. ${ }^{9,10}$ Grossly, osteoblastomas are often within cortex or cancellous bone. The lesion is red or purple due to its hemorrhagic nature, granular in appearance, and has cystic components. ${ }^{9}$

Histologically, osteoblastomas are comprised of wellvascularized connective tissue, with dilated capillaries and active production of osteoid and woven bone.

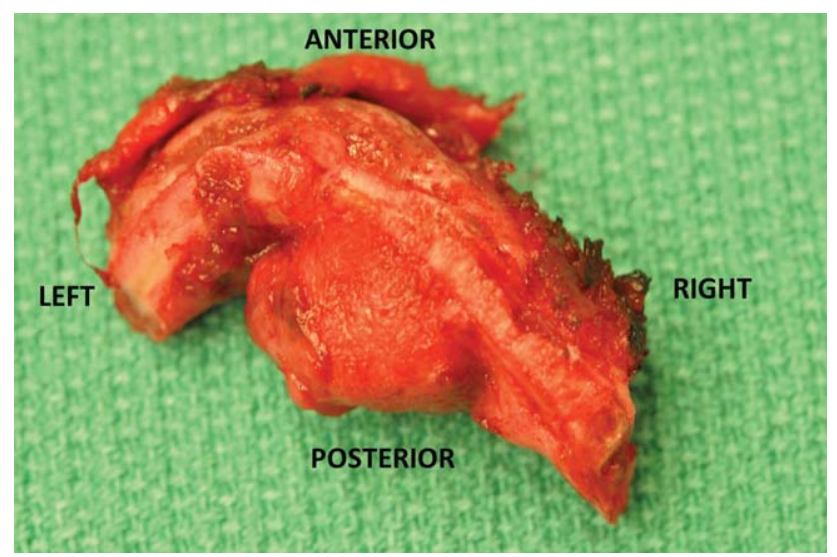

Fig. 6. Gross specimen from partial laryngectomy is a curved segment of thyroid cartilage with attached connective tissue measuring $3.1 \times 1.5 \times 1.4 \mathrm{~cm}$. Not visualized is the $1.5 \times 1.4 \times 1.1$ $\mathrm{cm}$ intracartilaginous mass, with well-circumscribed borders located $0.5 \mathrm{~cm}$ from the nearest peripheral margin and less than $0.1 \mathrm{~cm}$ from the deep margin.

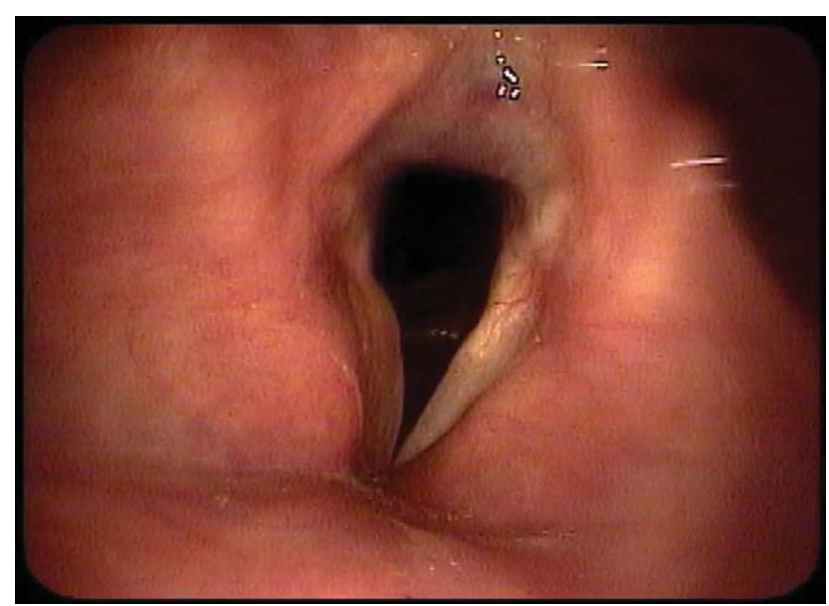

Fig. 7. Still image taken from in-office laryngeal videostroboscopy 1 week postoperatively showing mild edema in the right vocal fold. Vocal fold mobility was normal bilaterally.

Histology alone cannot distinguish osteoblastoma from osteoid osteoma. However, it can distinguish osteoblastoma from osteosarcoma as osteoblastoma will typically have the following characteristics: lack of nuclear atypia, mineralization of osteoid with a single rim of plump osteoblasts, low mitotic activity, round to oval regular nuclei, and a sharp, noninfiltrative margin. ${ }^{1,9}$ The most mature portion of an osteoblastoma will be at the periphery, with a sharp interface between normal bone and tumor. Rare aggressive osteoblastomas may contain focal areas of large cells with bizarre, atypical, hyperchromatic nuclei or chondroid cells, making those areas indistinguishable from osteosarcomas. However, even these aggressive lesions will lack significant mitotic activity, necrosis, malignant cartilage, or infiltration of the interface between tumor and normal bone. ${ }^{9,10}$ Chondrosarcoma will present with bone invasion presenting as calcification centers within cartilage, infiltrative growth,

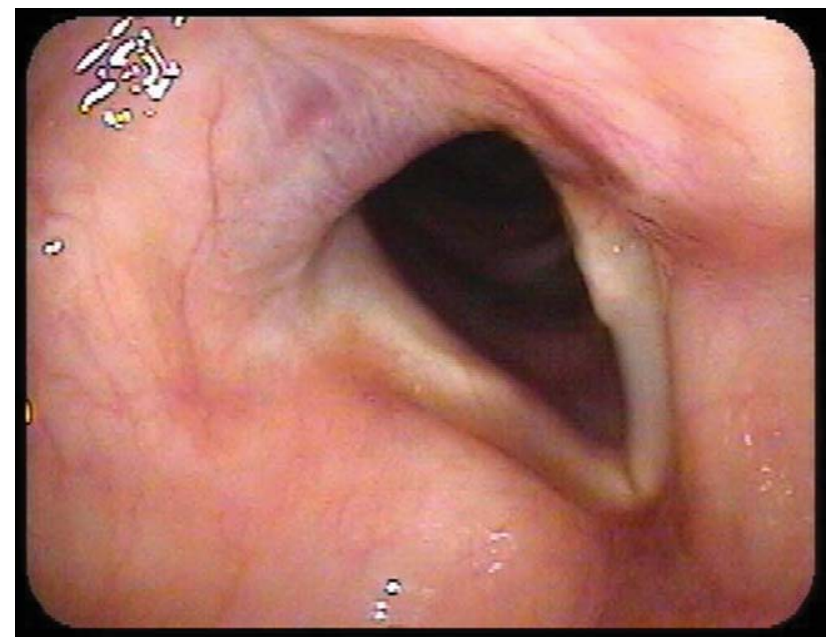

Fig. 8. Still image taken from in-office laryngeal videostroboscopy 7 weeks postoperatively. The right vocal fold edema had nearly completely resolved. 
increased cellularity, nuclear atypia, loss of normal architecture, and increased nuclear-to-cytoplasmic ratio. ${ }^{8}$ As with any tumor, large biopsy specimens are critical to adequately assess the tumor type and grade.

Cure is typically achieved by complete en bloc resection of the osteoblastoma. If en bloc resection is not possible and marginal or curettage resection is performed, local recurrence rates range from $10 \%$ to $20 \%$. Local recurrence is a risk factor for malignant transformation. Aggressive osteoblastomas have a higher rate of local recurrence, but neither conventional nor aggressive osteoblastomas have been reported to metastasize. ${ }^{9,10}$

\section{CONCLUSION}

Osteoblastoma of the laryngeal cartilage is a rare occurrence. Radiology alone cannot be used to distinguish osteoblastoma from other boney tumors; tissue biopsy is necessary for definitive diagnosis. Because of our patient's history of professional voice use, treatment required a tailored operation. Her surgery included elements of partial laryngectomy, as if for cancer, and careful dissection and maintenance of vital endolaryngeal soft tissues that would normally be resected en bloc with cartilage when treating for malignancy. We believe that low-grade laryngeal chondrosarcomas or osteosarcomas could be approached in a similar manner.

\section{BIBLIOGRAPHY}

1. Dellmann A, Schroeder H-G, Donhuijsen K. Destructive tumours of the larynx mimicking osteosarcoma: two cases of osteoblastoma with organsaving resection and follow-up. Eur Arch Otorhinolaryngol 2010;267:151154.

2. Kaliteevsky PF, Korolkova IA. A case of localization of osteoblastoclastoma in the larynx. Vestn Otorinolaringol 1966;28:69-71.

3. Agarwala R, Graham J, Panella JS. CT and MRI of aggressive osteoblastoma of thyroid cartilage. J Comput Assist Tomogr 1996;20:116-118.

4. Ledeboer Q, Kerrebijn J, Govaere F, den Bakker MA. Osteoblastoma of the larynx; a case report. Int J Surg Pathol 2005;13:365:367.

5. Urbanczyk H, Miszczyk L. Aggressive osteoblastoma of the larynx: case report. Otolaryngol Pol 2006;60:227-228

6. Mirbagherie N, Galloway S, Iseli TA, Lyons BM. Extraosseous osteoblastoma of the larynx presenting with acute airway obstruction. $J$ Laryngol Otol 2008;122:1265-1268.

7. Rosenberg A. Bones, Joints, and soft tissue tumors. In: Kumar V, ed. Robbins and Cotran pathologic basis of disease, 8th edition. Philadelphia, PA: Saunders Elsevier; 2010:1224-7.

8. Thompson LDR. Malignant neoplasms of the larynx, hypopharynx, and trachea. In: Thompson, LDR, ed. Head and Neck Pathology. Philadelphia, PA: Churchill Livingstone Elsevier; 2006:80-84.

9. El-Mofty SK. Bone lesions. In: Gnepp, DR, ed. Diagnostic Surgical Pathology of the Head and Neck. Philadelphia, PA: Saunders Elsevier; 2009:732-739.

10. Atesok KI, Alman BA, Schemitsch EH, Peyser A, Mankin H. Osteoid osteoma and osteoblastoma. J Am Acad Orthop Surg 2011;19:678-689. 